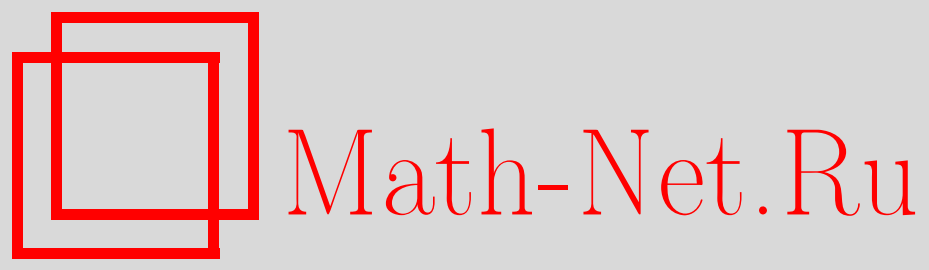

Я. Г. Синай, А. Б. Сошников, Уточнение полукругового закона Вигнера в окрестности края спектра для случайных симметричных матриц, Функи. анализ и его прил., 1998, том 32, выпуск $2,56-79$

DOI: https://doi.org/10.4213/faa411

Использование Общероссийского математического портала Math$\mathrm{Net.Ru}$ подразумевает, что вы прочитали и согласны с пользовательским соглашением

http://www . mathnet.ru/rus/agreement

Параметры загрузки:

IP: 34.227 .88 .159

26 апреля 2023 г., 15:51:43

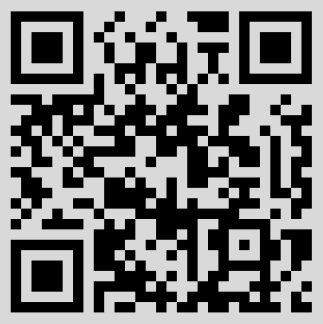


Функииональный анализ и его приложения

1998, т. 32, вып. 2, с. 56-79

УДК 519.2

\section{Уточнение полукругового закона Вигнера в окрестности края спектра для случайных симметричных матриц}

(c) 1998. Я. Г. Синай, А. Б. Сошников

\section{$\S 1$. Введение и формулировка результатов}

Мы рассматриваем ансамбль Вигнера $n$-мерных вещественных случайных симметричных матриц $A_{n}=\left\|a_{i j}\right\|$, где $a_{i j}=a_{j i}=\xi_{i j} / \sqrt{n}, 1 \leqslant i \leqslant j \leqslant n$, и $\xi_{i j}$ образуют набор независимых вещественных случайных величин. Мы предполагаем, что

(i) случайные величины $\xi_{i j}$ имеют симметричные законы распределения и

$$
\mathbf{E}\left(\xi_{i j}\right)^{2}=1 / 4 \text { при } i<j \quad \text { и } \quad \mathbf{E}\left(\xi_{i i}\right)^{2} \leqslant \text { const ; }
$$

здесь и далее $\mathbf{E}$ есть знак математического ожидания, a const - это числа, не зависящие от $n$.

(ii) все моменты случайных величин $\xi_{i j}$ конечны и допускают оценку

$$
\mathbf{E}\left(\xi_{i j}\right)^{2 m} \leqslant(\text { const } \cdot m)^{m} ;
$$

это означает, что распределения случайных величин $\xi_{i j}$ убывают не медленнее, чем гауссовские.

Обозначим через $\lambda_{k}, k=1, \ldots, n$, собственные значения матрицы $A$. Предельная теорема Вигнера (см. $[1,2])$ утверждает, что эмпирическая функция распределения чисел $\lambda_{k}$

$$
N_{n}(\lambda)=\frac{1}{n} \#\left\{k: \lambda_{k}<\lambda\right\}
$$

сходится при $n \rightarrow \infty$ по вероятности к распределению, отвечающему полукруговому закону Вигнера

$$
\lim _{n \rightarrow \infty} N_{n}(\lambda)=\int_{-\infty}^{\lambda} \rho(u) d u,
$$

где

$$
\rho(u)= \begin{cases}0 & \text { при } u>1 \text { и } u<-1, \\ \frac{2}{\pi} \sqrt{1-u^{2}} & \text { при }-1 \leqslant u \leqslant 1 .\end{cases}
$$

В дальнейшем этот результат был усилен В. А. Марченко и Л. А. Пастуром, Л. Арнольдом, К. Вахтером, В. Л. Гирко и др. [3-13].

* Исследования первого автора поддержаны РФФИ (грант 96-01-0037) и NSF (грант DMS-9706794), второго — NSF (грант DMS-9304580). 
Рассмотрим окрестность $O_{n}$ правого конца спектра $\lambda=1$ размера $r_{n}$, где $r_{n} n^{2 / 3} \rightarrow \infty$ при $n \rightarrow \infty$. Например, можно выбрать $r_{n} \sim$ const $/ n^{\gamma}, \gamma<2 / 3$. Формально применяя полукруговой закон, получим, что число собственных значений в $O_{n}$ ведет себя как const $\cdot r_{n}^{3 / 2} n$. Перенормируем собственные значения, положив

$$
\lambda_{k}=1-\theta_{k} r_{n}
$$

и поместим в каждую точку $\theta_{k}$ массу

$$
\mu_{n}\left(\theta_{k}\right)=\frac{1}{n r_{n}^{3 / 2}}
$$

Мы получаем при этом меру $\mu_{n}$ на прямой, для которой $\mu_{n}\left(\mathbb{R}^{1}\right)=r_{n}^{-3 / 2}$. Основной результат этой работы составляет следующая теорема.

ТЕОРЕМА 1 (ОСНОВНАЯ ТЕОРЕМА). При $n \rightarrow \infty$ меры $\mu_{n}$ слабо сходятся по вероятности на каждом конечном отрезке $\kappa$ мере $\mu$, сосредоточенной на полупрямой $\mathbb{R}^{+}$и абсолютно непрерьвной относительно мерь Лебега, для которой плотность $d \mu(x) / d x$ имеет вид $(2 \sqrt{2} / \pi) \sqrt{x}$ при $x \geqslant 0$ и равна нулю при $x<0$.

Если $r_{n}>n^{\varepsilon-2 / 3}$ при некотором $\varepsilon>0$, то меры $\mu_{n}$ слабо сходятся $\kappa \mu$ с вероятностью 1 .

Аналогичная теорема верна для собственных значений в окрестности левой границы спектра $\lambda=-1$.

Из основной теоремы вытекает ряд следствий. Пусть $\lambda_{\max }\left(A_{n}\right)$ - максимальное собственное значение матрицы $A_{n}$. Запишем его в следующем виде:

$$
\lambda_{\max }\left(A_{n}\right)=1+\theta_{\max }\left(A_{n}\right) n^{-2 / 3} .
$$

СЛЕДСТВИЕ 1. Случайные величины $\theta_{\max }\left(A_{n}\right)$ равномерно ограничены по вероятности, т.е. для любого $\varepsilon>0$ можно найти такое число $M$, что

$$
\mathbf{P}\left\{\theta_{\max }\left(A_{n}\right) \leqslant M\right\} \leqslant \varepsilon
$$

при всех достаточно больиих $n$.

СЛЕдСТВИЕ 2. Пусть $\nu^{+}\left(A_{n}, x\right)$ - число собственных значений, лежаиих правее $1+n^{-2 / 3} x$, где $x$ - произвольное вещественное число. Тогда

$$
\mathbf{E} \nu^{+}\left(A_{n}, x\right) \leqslant \operatorname{const}(x) .
$$

Из основной теоремы вытекает также, что в окрестности $O_{n}$ число собственных значений растет как $n r_{n}^{3 / 2}$. Поэтому среднее расстояние между собственными значениями в $O_{n}$ убывает как $n^{-1} r_{n}^{-1 / 2}$.

Основная теорема вытекает из следующей теоремы.

TEOPEMA 2. Пусть $p_{n} \rightarrow \infty$ npu $n \rightarrow \infty$ mak, иmo $p_{n}=o\left(n^{2 / 3}\right)$. Тогдa

$$
\mathbf{E}\left(\operatorname{Tr} A_{n}^{p_{n}}\right)= \begin{cases}2^{3 / 2} n\left(\pi p_{n}^{3}\right)^{-1 / 2}(1+o(1)), & \text { если } p_{n} \text { четно } \\ 0, & \text { если } p_{n} \text { чечетно }\end{cases}
$$


и распределение $\operatorname{Tr} A_{n}^{p_{n}}-\mathbf{E}\left(\operatorname{Tr} A_{n}^{p_{n}}\right)$ слабо сходитсл $к$ нормальному закону $N(0,1 / \pi)$.

Теорема 2 представляет собой усиление аналогичной теоремы, доказанной нами в [14] для $p_{n}=o\left(n^{1 / 2}\right)$.

ЗАмЕчАНИЕ 1. Можно сформулировать и многомерный вариант теоремы 2. Пусть

$$
q_{n}^{(i)}=c^{(i)} p_{n} \cdot(1+o(1)), \quad i=1, \ldots, l,
$$

где $c^{(i)}$ - положительные константы, и $q_{n}^{\left(i_{1}\right)}-q_{n}^{\left(i_{2}\right)}$ четны. Тогда

$$
\lim _{n \rightarrow \infty} \operatorname{Cov}\left(\operatorname{Tr} A_{n}^{q_{n}^{\left(i_{1}\right)}}, \operatorname{Tr} A_{n}^{q_{n}^{\left(i_{2}\right)}}\right)=\frac{2}{\pi} \frac{\sqrt{c_{i_{1}} c_{i_{2}}}}{c_{i_{1}}+c_{i_{2}}}, \quad 1 \leqslant i_{1}, i_{2} \leqslant l,
$$

и совместное распределение случайных величин $\operatorname{Tr} A_{n}^{q_{n}^{(i)}}-\mathbf{E}\left(\operatorname{Tr} A_{n}^{q_{n}^{(i)}}\right)$ сходится к многомерному нормальному распределению. Если же выбрать $q_{n}^{\left(j_{1}\right)}$, $q_{n}^{\left(j_{2}\right)}$ так, что $q_{n}^{\left(j_{1}\right)}-q_{n}^{\left(j_{2}\right)}$ нечетно, то центрированные следы асимптотически независимы при $n \rightarrow \infty$.

Теорема 2 доказывается в $\S \S 4,5$. В $\S 2$, предполагая теорему 2 доказанной, мы выводим из нее основную теорему и следствия.

Результаты статьи справедливы также для ансамбля Вигнера комплексных самосопряженных матриц. Это и другие замечания обсуждаются в $\S 3$.

\section{§2. Вывод основной теоремы и следствий из теоремы 2}

Для доказательства основной теоремы достаточно установить сходимость преобразований Лапласа мер $\mu_{n}$, т. е. показать, что

$$
\int_{-\infty}^{\infty} e^{-c \theta} d \mu_{n}(\theta)=\frac{1}{n r_{n}^{3 / 2}} \sum_{k=1}^{n} e^{-c \theta_{k}} \underset{n \rightarrow \infty}{\stackrel{\mathbf{P}}{\longrightarrow}} \int_{0}^{+\infty} e^{-c \theta} \frac{2 \sqrt{2}}{\pi} \sqrt{\theta} d \theta=\sqrt{\frac{2}{\pi c^{3}}},
$$

где $c>0$.

Положим $s_{n}=\left[(c / 2) r_{n}^{-1}\right]$ и $p_{n}=2 s_{n}$ и введем следующую перенормировку для положительных и отрицательных собственных значений:

$$
\begin{aligned}
\lambda_{k}=1-\theta_{k} r_{n}, & & \lambda_{k} \geqslant 0, \\
\lambda_{j}=-1+\tau_{j} r_{n}, & & \lambda_{j}<0 .
\end{aligned}
$$

Согласно теореме $2, n^{-1} r_{n}^{-3 / 2} \operatorname{Tr} A^{2 s_{n}}$ сходится по вероятности к

$$
\lim _{n \rightarrow \infty} \frac{1}{n r_{n}^{3 / 2}} \mathbf{E}\left(\operatorname{Tr} A^{2 s_{n}}\right)=\lim _{n \rightarrow \infty} \frac{1}{n r_{n}^{3 / 2}} \frac{n}{\sqrt{\pi s_{n}^{3}}}=\frac{2 \sqrt{2}}{\sqrt{\pi c^{3}}},
$$

a $n^{-1} r_{n}^{-3 / 2} \operatorname{Tr} A^{2 s_{n}+1}$ сходится по вероятности к нулю.

Принимая во внимание (2.2), получаем, что

$$
\operatorname{Tr} A^{2 s_{n}}=\sum_{k}\left(1-r_{n} \theta_{k}\right)^{2\left[(c / 2) r_{n}^{-1}\right]}+\sum_{j}\left(1-\tau_{j} r_{n}\right)^{2\left[(c / 2) r_{n}^{-1}\right]}
$$


И

$$
\operatorname{Tr} A^{2 s_{n}+1}=\sum_{k}\left(1-r_{n} \theta_{k}\right)^{2\left[(c / 2) r_{n}^{-1}\right]+1}-\sum_{j}\left(1-\tau_{j} r_{n}\right)^{2\left[(c / 2) r_{n}^{-1}\right]+1}
$$

Заметим, что если $\left|\theta_{k}\right|<r_{n}^{-1 / 3},\left|\tau_{j}\right|<r_{n}^{-1 / 3}$, то соответствующие слагаемые в $(2.3),(2.4)$ суть соответственно $e^{-c \theta_{j}}\left(1+o\left(r_{n}^{1 / 3}\right)\right), e^{-c \tau_{j}}\left(1+o\left(r_{n}^{1 / 3}\right)\right)$. С другой стороны, из оценки в теореме 2 математического ожидания $\mathbf{E}\left(\operatorname{Tr} A^{4 s_{n}}\right)$ следует, что подсуммы в $(2.3),(2.4)$ по $\theta_{k}, \tau_{j}$, таким, что

$$
\left|\theta_{k}\right|>r_{n}^{-1 / 3}, \quad\left|\tau_{j}\right|>r_{n}^{-1 / 3},
$$

стремятся по вероятности к нулю. Также будут стремиться к нулю (по вероятности) подсуммы по таким $\theta_{k}, \tau_{j}$ линейных статистик $\sum_{k} e^{-c \theta_{k}}, \sum_{j} e^{-c \tau_{j}}$. Из этих рассуждений и формул (2.3), (2.4) вытекает, что

$$
\left(\operatorname{Tr} A^{2 s_{n}}+\operatorname{Tr} A^{2 s_{n}+1}-2 \sum_{k} e^{-c \theta_{k}}\right) \frac{1}{n r_{n}^{3 / 2}} \underset{n \rightarrow \infty}{\stackrel{\mathbf{P}}{\longrightarrow}} 0
$$

и, как следствие,

$$
\frac{1}{n r_{n}^{3 / 2}} \sum_{k} e^{-c \theta_{k}} \underset{n \rightarrow \infty}{\stackrel{\mathbf{P}}{\longrightarrow}} \sqrt{\frac{2}{\pi c^{3}}}
$$

Если $r_{n}=n^{-\gamma}, \gamma<2 / 3$, то из теоремы 2 следует, что нормированные следы $n^{-1} r_{n}^{-3 / 2} \operatorname{Tr} A^{2 s_{n}}, n^{-1} r_{n}^{-3 / 2} \operatorname{Tr} A^{2 s_{n}+1}$ сходятся к неслучайным пределам с вероятностью 1 , и, таким образом, сходимость мер $\mu_{n}$ в основной теореме также имеет место с вероятностью 1. Из теоремы 2 также следует, что флуктуации случайных величин $\sum_{k} e^{-c \theta_{k}}$ имеют порядок константы и сходятся по распределению к нормальному закону $N(0,1 / 2 \pi)$ при $n \rightarrow \infty$, если $r_{n} \ll n^{-2 / 5}$.

Ниже мы доказываем следствия 1 и 2.

Запишем максимальное собственное значение в виде $\lambda_{\max }=1+\theta_{\max } n^{-2 / 3}$. Предположим, что существуют последовательности $n_{i} \rightarrow \infty, L_{i} \rightarrow \infty$ и такое $\varepsilon>0$, что

$$
\mathbf{P}\left\{\lambda_{\max }\left(A_{n_{i}}\right)>1+n_{i}^{-2 / 3} L_{i}\right\}>\varepsilon>0 .
$$

Рассмотрим $\operatorname{Tr} A_{n_{i}}^{p_{n_{i}}}$, где $P_{n_{i}}=2\left[n^{2 / 3} / L_{i}^{1 / 2}\right]$ (для удобства можно всегда предполагать, что $\left.L_{i} \ll n^{2 / 3}\right)$, и применим теорему 2. Тогда

$$
\mathbf{E}\left(\operatorname{Tr} A_{n_{i}}^{p_{n_{i}}}\right) \leqslant \frac{2}{\sqrt{\pi}} L_{i}^{3 / 2}
$$

при достаточно больших $n_{i}$.

С другой стороны, из неравенства (2.5) вытекало бы, что

$$
\mathbf{E}\left(\operatorname{Tr} A_{n_{i}}^{p_{n_{i}}}\right)>\mathbf{E} \lambda_{\max }\left(A_{n_{i}}\right)^{p_{n_{i}}}>\varepsilon\left(1+n_{i}^{-2 / 3} L_{i}\right)^{2\left[n_{i}^{2 / 3} / \sqrt{L_{i}}\right]} \geqslant \varepsilon e^{\sqrt{L_{i}}} .
$$

При достаточно больших $L_{i}$ последнее неравенство противоречит (2.6). Следствие 1 доказано. 
ДокАЗАТЕЛЬСТво СЛЕДСТВИя 2. Следствие 2 также доказывается от противного. Предположим, что существуют такие последовательности $n_{i} \rightarrow \infty$, $L_{i} \rightarrow \infty$, что

$$
\mathbf{E} \nu^{+}\left(A_{n_{i}}, x\right)>L_{i},
$$

где $\nu^{+}\left(A_{n}, x\right)$ - число собственных значений, лежащих правее $1+n^{-2 / 3} x$, где $x>0$ произвольно. Для того чтобы получить противоречие с $(2.8)$,

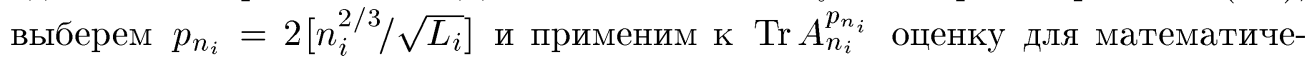
ского ожидания из теоремы 2. Получаем, что при достаточно больших $n_{i}$ $\mathbf{E}\left(\operatorname{Tr} A_{n_{i}}^{p_{n_{i}}}\right) \leqslant(2 / \sqrt{\pi}) L_{i}^{3 / 4}$. С другой стороны,

$$
\mathbf{E} \nu^{+}\left(A_{n_{i}}, x\right) \leqslant \frac{\mathbf{E}\left(\operatorname{Tr} A_{n_{i}}^{p_{n_{i}}}\right)}{\left(1-x n_{i}^{-2 / 3}\right)^{2\left[n_{i}^{2 / 3} / L_{i}^{2 / 3}\right]}} \leqslant 2 \mathbf{E}\left(\operatorname{Tr} A_{n_{i}}^{p_{n_{i}}}\right) \leqslant \frac{4}{\sqrt{\pi}} L_{i}^{3 / 4}
$$

при достаточно больших $n_{i}$, что противоречит предположению (2.8), если выбрать $L_{i}$ достаточно большим. Следствие 2 доказано.

\section{§3. Дополнительные замечания}

1. Аналогичные результаты справедливы для ансамбля Вигнера комплексных самосопряженных матриц $A_{n}=\left\{a_{k j}\right\}_{1 \leqslant k, j \leqslant n}$, где

$$
a_{k j}=\overline{a_{j k}}=\frac{\operatorname{Re} \xi_{k j}+i \operatorname{Im} \xi_{k j}}{n^{1 / 2}}, \quad 1 \leqslant k<j \leqslant n, \quad a_{k k}=\frac{\xi_{k k}}{n^{1 / 2}}, \quad k=1, \ldots, n,
$$

причем $\mathbf{E}\left|\xi_{k j}\right|^{2}=1 / 4$ при $k<j$ и $\mathbf{E} \xi_{k k}^{2} \leqslant$ const, и для ансамбля положительно определенных матриц $B_{n} B_{n}^{*}$ в окрестности правого края спектра в предположении, что все матричные элементы $b_{i j}=n^{-1 / 2} \eta_{i j}$ являются независимыми случайными величинами.

2. Если матричные элементы являются гауссовскими случайными величинами, для многих характеристик локального распределения собственных значений известны явные формулы (см. $[20,16])$. В частности, известно, что распределение точечного случайного поля $\left\{x_{1}, \ldots, x_{n}\right\}$, заданного формулой

$$
\lambda_{i}=1+n^{-2 / 3} x_{i}, \quad i=1, \ldots, n,
$$

имеет предел при $n \rightarrow \infty$. Предельное точечное случайное поле, которое отвечает за локальное распределение собственных значений у края спектра, определяется своими $k$-точечными корелляционными функциями, $k=$ $1,2, \ldots$ В случае ансамбля эрмитовых матриц, таких, что

$$
\operatorname{Re} \xi_{k j} \sim N\left(0, \frac{1}{8}\right), \quad \operatorname{Im} \xi_{k j} \sim N\left(0, \frac{1}{8}\right), \quad 1 \leqslant k<j \leqslant n, \quad \xi_{k k} \sim N\left(0, \frac{1}{4}\right),
$$

известного в литературе по случайным матрицам [20] как гауссовский унитарный ансамбль, $k$-точечные корреляционные функции представляют собой определитель $k$-мерных матриц с ядром

$$
K(x, y)=\frac{A(x) A^{\prime}(y)-A^{\prime}(x) A(y)}{x-y},
$$


где $A(x)$ - функция Эйри, и

$$
\rho_{k}\left(y_{1}, \ldots, y_{k}\right)=\operatorname{det}\left(K\left(y_{i}, y_{j}\right)\right)_{1 \leqslant i, j \leqslant k}
$$

(cм. [16, 17]).

Интересно, что для ансамбля симметричных матриц с гауссовскими компонентами

$$
\xi_{i j} \sim N\left(0, \frac{1}{4}\right), \quad 1 \leqslant i<j \leqslant n, \quad \xi_{i i} \sim N\left(0, \frac{1}{2}\right), \quad 1 \leqslant i \leqslant n
$$

(гауссовский ортогональный ансамбль) локальное распределение собственных значений отлично от (3.2) (см. [15, 20]). И в том, и в другом случае точечное случайное поле сгушается при $x \rightarrow-\infty$, т.е. расстояние между ближайшими собственными значениями стремится к нулю, причем спектральная плотность $\rho_{1}(x)$ асимптотически эквивалентна плотности Вигнера при $x \rightarrow-\infty$. Это указывает на справедливость (локального) закона Вигнера в окрестности конца спектра на любом масштабе, большем чем $n^{-2 / 3}$. Основная теорема данной статьи утверждает, что такой результат справедлив и для произвольного вигнеровского ансамбля. А именно, рассмотрим точечное случайное поле $\left\{x_{1}, \ldots, x_{n}\right\}$, определяемое формулой $(3.1)$, выберем $R_{n} \rightarrow$ $+\infty$ так, что $R_{n} \ll n^{2 / 3}$, и подсчитаем число точек $x_{i}$, лежащих правее $-R_{n}$ : $N_{n}\left(-R_{n}\right)=\#\left\{x_{i}>-R_{n}\right\}$. По определению $N_{n}\left(-R_{n}\right)=\#\left\{\lambda_{i}>1-n^{-2 / 3} R_{n}\right\}$. Тогда вследствие основной теоремы в главном порядке величина $N_{n}\left(-R_{n}\right)$ неслучайна и равна

$$
n \int_{1-n^{-2 / 3} R_{n}}^{1} \frac{2}{\pi} \sqrt{1-t^{2}} d t \cdot(1+o(1)) .
$$

Вопрос о порядке флуктуации случайной величины $N_{n}\left(-R_{n}\right)$ пока остается открытым. Естественно предположить, что по аналогии со случаем локального распределения собственных значений внутри спектра для гауссовских ансамблей (см. [21]) эта величина будет иметь порядок $\sqrt{\log \left(R_{n}\right)}$. Частичным подтверждением этой гипотезы является следствие из теоремы 2, утверждающее, что для сглаженных считающих функщий $\sum_{i} e^{c x_{i} / R_{n}}, c>0$, при $R_{n} \ll n^{2 / 3-2 / 5}$ флуктуации имеют порядок константы и центрированные линейные статистики

$$
\sum_{i} e^{c x_{i} / R_{n}}-\mathbf{E}\left(\sum_{i} e^{c x_{i} / R_{n}}\right)
$$

сходятся по распределению к нормальному закону $N\left(0, \frac{1}{2 \pi}\right)$.

3. Первые результаты про следы больших степеней вигнеровских матриц принадлежат Фюреди и Комлозу [18], а также Буте де Монвель и Щербине [19]. В частности, Фюреди и Комлоз рассмотрели случай равномерно ограниченных случайных величин $\xi_{i j}$ (не обязательно с симметричным законом распределения) и доказали формулу для главного порядка математического ожидания $\mathbf{E}\left(\operatorname{Tr} A^{P_{n}}\right)$ при $p_{n} \ll n^{1 / 6}$. Отметим, что техника нашей статьи может быть обобщена на случай несимметрично распределенных случайных величин $\xi_{i j}$. При этом возникает необходимость изучать пути, проходящие некоторое ребро нечетное (большее чем один) число раз (см. $\S \S 4,5)$. 
При $p_{n} \ll n^{1 / 2}$ основная теорема и теорема 2 остаются справедливыми и для несимметрично распределенных $\xi_{i j}$. Более того, при $p_{n} \ll n^{1 / 2}$ можно ослабить условие (1.1) на рост моментов случайных величин $\xi_{i j}$, потребовав, чтобы функции распределения $\xi_{i j}$ убывали на бесконечности не медленнее, чем экспоненциально, т. е. заменив (1.1) на

$$
\mathbf{E}\left(\xi_{i j}\right)^{2 k} \leqslant(\text { const } \cdot k)^{2 k} .
$$

\section{§4. Математическое ожидание $\mathbf{E}\left(\operatorname{Tr} A_{n}^{p_{n}}\right)$}

Теорема 2 доказывается методом моментов. Для этого необходимо показать, что моменты случайной величины $\operatorname{Tr} A_{n}^{p_{n}}-\mathbf{E}\left(\operatorname{Tr} A_{n}^{p_{n}}\right)$ сходятся к соответствующим моментам нормального распределения $N\left(0, \frac{1}{\pi}\right)$. В этом параграфе оценивается главный порядок математического ожидания $\mathbf{E}\left(\operatorname{Tr} A_{n}^{p_{n}}\right)$. Ясно, что

$$
\mathbf{E}\left(\operatorname{Tr} A_{n}^{2 s_{n}}\right)=\frac{1}{n^{s_{n}}} \sum_{\mathscr{P}} \mathbf{E} \xi_{i_{0} i_{1}} \cdot \xi_{i_{1} i_{2}} \cdot \ldots \cdot \xi_{i_{2 s_{n}-1}, i_{0}} .
$$

Суммирование в (4.1) производится по всем замкнутым путям $\mathscr{P}=\left\{i_{0}, i_{1}\right.$, $\left.\ldots, i_{2 s_{n}-1}, i_{0}\right\}$ с выделенным началом на множестве $\{1, \ldots, n\}$. Удобно считать множество вершин $\{1, \ldots, n\}$ неориентированным графом, в котором любые две вершины соединены неупорядоченным ребром. В силу симметрии распределения случайных величин $\xi_{i j}$ ненулевой вклад в (4.1) вносят пути, в которых каждое ребро встречается четное число раз. Такие пути будем называть четными. В дальнейшем только четные и рассматриваются.

ОПРЕДЕлЕНИЕ 1. Момент времени $k$ называется отмеченным, если (неупорядоченное) ребро $\left\{i_{k-1}, i_{k}\right\}$ встретилось до момента $k$ включительно нечетное число раз. Остальные моменты времени называются неотмеченньлми.

У четных путей число отмеченных моментов времени равно числу неотмеченных моментов и равно $s_{n}=p_{n} / 2$. Каждому пути $\mathscr{P}$ сопоставим траекторию случайного блуждания на положительной полуоси $X=\{x(0), x(1), \ldots$, $\left.x\left(2 s_{n}\right)\right\}$, где $x(0)=0$ и $x(k)-x(k-1)$ равно 1 или -1 в зависимости от того, будет $k$-й момент отмеченным или неотмеченным. Очевидно, что $x(t) \geqslant 0$ при всех $0 \leqslant t \leqslant p_{n}$ и для четных путей $x\left(p_{n}\right)=0$.

ОПредЕлениЕ 2. Четный путь $\mathscr{P}$ называется путем без самопересечений, если не существует двух отмеченных моментов времени $k^{\prime}, k^{\prime \prime}$, для которых $i_{k^{\prime}}=i_{k^{\prime \prime}}$. Пути без самопересечений имеют следующую структуру. Сначала идет серия отмеченных моментов, при которых путь проходит соответствующее число различных вершин, равное длине серии. Затем следует серия неотмеченных моментов, при которых путь проходит часть тех же вершин в обратном порядке, потом появляется новая серия отмеченных моментов времени и отвечающая им серия вершин и т.д. Существенно, что в случае путей без самопересечений траектория в неотмеченные моменты времени определена однозначно. Каждое ребро проходится в таких путях два раза. 
Подсумма в сумме (4.1) по путям без самопересечений равна

$$
Z(0)=\frac{1}{n^{s_{n}}} \sum_{\substack{\mathscr{P} \text { без } \\ \text { самоперес. }}} \mathbf{E} \xi_{i_{0} i_{1}} \cdots \xi_{i_{2 s_{n}-1} i_{0}}=\frac{1}{n^{s_{n}}} \frac{1}{4^{s_{n}}} \frac{\left(2 s_{n}\right) ! n(n-1) \cdots\left(n-s_{n}\right)}{s_{n} !\left(s_{n}+1\right) !} .
$$

Здесь $\left(2 s_{n}\right) ! /\left(s_{n} !\left(s_{n}+1\right) !\right)$ есть число траекторий длины $2 s_{n}$ простейшего случайного блуждания на неотрицательной полупрямой, для которых $x(0)=$ $x\left(2 s_{n}\right)=0$, а произведение $n(n-1) \cdots\left(n-s_{n}\right)$ задает число способов выбора начальной вершины и вершин в отмеченные моменты времени. В [14] было показано, что при $s_{n}=o\left(n^{1 / 2}\right)$ главный вклад в $\mathbf{E}\left(\operatorname{Tr} A_{n}^{2 s_{n}}\right)$ вносится именно путями без самопересечений, т.е.

$$
\mathbf{E}\left(\operatorname{Tr} A_{n}^{2 s_{n}}\right)=Z(0) \cdot(1+o(1)) .
$$

В общем случае это уже не так, и нам необходимо исследовать статистику самопересечений.

ОПРЕДЕЛЕНИЕ 3. Отмеченный момент времени $m$ называется моментом самопересечения, если найдется отмеченный момент времени $m^{\prime}<m$, такой, что $i_{m^{\prime}}=i_{m}$.

ОПРЕДЕЛЕНИЕ 4. Вершина $i$ называется вершиной простого (тройного, четверного, и т.д.) пересечения, если сушествует ровно два (три, четыре и т.д.) отмеченных момента времени $i_{m}$, таких, что $i_{m}=i$.

Согласно определению 4 , все вершины разбиты на $s_{n}+1$ непересекающихся подмножеств:

$$
\{1, \ldots, n\}=\bigsqcup_{k=0}^{s_{n}} N_{k}
$$

где $N_{k}$ - подмножество вершин $k$-кратного пересечения, т. е., переформулируя определение 4, мы говорим, что вершина $i$ принадлежит классу $N_{k}$, $k \geqslant 0$, если найдется ровно $k$ отмеченных моментов времени $m_{1}, \ldots, m_{k}$, таких, что $i_{m_{j}}=i, j=1, \ldots, k$. Все, кроме одной, вершины из $N_{0}$ не принадлежат пути $\mathscr{P}$. Единственным исключением может быть начало пути $i_{0}$ при условии, что оно не посещалось в отмеченные моменты времени на промежуточных шагах пути. Положим $n_{k}=\#\left(N_{k}\right)$. Легко видеть, что

$$
\sum_{k=0}^{s_{n}} n_{k}=n, \quad \sum_{k=0}^{s_{n}} k n_{k}=s_{n}
$$

Мы будем называть Я尹 путем muna $\left(n_{0}, n_{1}, \ldots, n_{s_{n}}\right)$. Например, любой четный путь без самопересечений является путем типа $\left(n-s_{n}, s_{n}, 0, \ldots 0\right)$. В [14] было показано, что подсумма по путям типа $\left(n_{0}, n_{1}, \ldots, n_{s_{n}}\right)$ может быть оценена сверху величиной

$$
\frac{1}{n^{s_{n}}} \frac{n !}{n_{0} ! n_{1} ! \cdots n_{s_{n}} !} n \frac{\left(2 s_{n}\right) !}{s_{n} !\left(s_{n}+1\right) !} \frac{s_{n} !}{\prod_{k=1}^{s_{n}}(k !)^{n_{k}}} U\left(n_{0}, n_{1}, \ldots, n_{s_{n}}\right)
$$

где

$$
U\left(n_{0}, n_{1}, \ldots, n_{s_{n}}\right)=\max _{\substack{\mathscr{P} \text { типа } \\\left(n_{0}, n_{1}, \ldots, n_{s_{n}}\right)}} \mathbf{E}\left(\prod_{l=0}^{s_{n}} \xi_{i_{l} i_{l+1}}\right) W_{n}
$$


a $W_{n}$ - число способов выбора траектории пути в неотмеченные моменты времени при условии, что вершины в отмеченные моменты времени уже выбраны. Например, для путей без самопересечений $W_{n}=1$.

В [14] мы использовали следующую оценку для $U\left(n_{0}, n_{1}, \ldots, n_{s_{n}}\right)$ :

$$
U\left(n_{0}, n_{1}, \ldots, n_{s_{n}}\right) \leqslant\left(\frac{1}{4}\right)^{n_{1}} \prod_{k=2}^{s_{n}}\left(\mathrm{const}_{1} \cdot k\right)^{2 k n_{k}} .
$$

Эта оценка далека от оптимальной, если $n_{k}>0$ при больших $k$. Ниже, в лемме 1, мы показываем, что

$$
U\left(n_{0}, n_{1}, \ldots, n_{s_{n}}\right) \leqslant\left(\frac{1}{4}\right)^{n_{1}} \prod_{k=2}^{s_{n}}\left(\text { const }_{2} \cdot k\right)^{k n_{k}} .
$$

Выполняя суммирование по всем путям типа $\left(n_{0}, n_{1}, \ldots, n_{s_{n}}\right)$, таким, что

$$
\sum_{k=2}^{s_{n}} k n_{k}>10 \frac{s_{n}^{2}}{n}
$$

и используя оценки (4.4), (4.5), легко получить, что подсумма по таким путям есть $o(1)$ и, значит, мала по сравнению с устанавливаемой в теореме 2 величиной всей суммы

$$
\mathbf{E}\left(\operatorname{Tr} A_{n}^{2 s_{n}}\right)=\frac{n}{\sqrt{\pi s_{n}^{3}}}(1+o(1)) .
$$

В дальнейшем мы будем изучать сумму по путям, для которых всегда

$$
\sum_{k=2}^{s_{n}} k n_{k}<10 \frac{s_{n}^{2}}{n}
$$

Пусть $M=\sum_{k=2}^{s_{n}}(k-1) n_{k}$. Если путь $\mathscr{P}$ содержит только простые самопересечения, то $M=n_{2}$. В рассматриваемом нами случае $s_{n}=o\left(n^{2 / 3}\right)$, как мы покажем, именно пути с простыми самопересечениями вносят основной вклад в $\mathbf{E}\left(\operatorname{Tr} A_{n}^{2 s_{n}}\right)$. Обозначим сумму по путям с $M$ простыми самопересечениями через $Z(M)$. Справедливо следующее

ПРЕДЛОЖЕНИЕ 1.

$$
Z(M)=\frac{n}{\sqrt{\pi s_{n}^{3}}} e^{-s_{n}^{2} / 2 n} \frac{1}{M !}\left(\frac{s_{n}^{2}}{2 n}\right)^{M}(1+o(1))
$$

равномерно по $0 \leqslant M \leqslant 10 s_{n}^{2} / n$.

ЗАмЕчАНИЕ 2. Вследствие формулы (4.7) для типичных путей число самопересечений $M$ имеет порядок $s_{n}^{2} / n$ и равно $\left(s_{n}^{2} / 2 n\right)\left(1+O\left(\sqrt{n} / s_{n}\right)\right)$ при $s_{n} \gg \sqrt{n}$.

ДоКАЗАТЕЛЬСТВО ПРЕДЛОЖЕНИЯ 1. Подсчет суммы $Z(M)$ проведем следующим образом. В первую очередь выбираем $s_{n}=p_{n} / 2$ моментов времени, 
которые будут отмеченными. Обозначим отмеченные моменты времени в порядке возрастания: $0 \leqslant t_{1}<t_{2}<\cdots<t_{s_{n}}<2 s_{n}$. Напомним, что выбору отмеченных моментов времени отвечает траектория простейшего случайного блуждания $x(t), 0 \leqslant t \leqslant 2 s_{n}$, на положительной полуоси,

$$
\begin{gathered}
x(0)=x\left(2 s_{n}\right)=0, \\
x\left(t_{j}\right)-x\left(t_{j}-1\right)=1, \quad j=1, \ldots, s_{n}, \\
x(t)-x(t-1)=-1, \quad \text { если } t \neq t_{1}, \ldots, t_{s_{n}} .
\end{gathered}
$$

Затем мы выбираем $M$ моментов самопересечения $t_{j_{1}}, t_{j_{2}}, \ldots, t_{j_{M}}$ среди отмеченных моментов времени (т. е. выбираем индексы $j_{1}, \ldots, j_{M}$, такие, что $1 \leqslant j_{1}<j_{2}<\cdots<j_{M} \leqslant s_{n}$ ). После этого выбираем начало пути и вершины, появляющиеся в отмеченные моменты времени. Начальную точку мы выбираем $n$ способами, затем $n-1, n-2, \ldots, n-s_{n}+M$ способами последовательно выбираем вершины, появляющиеся в отмеченные моменты времени без самопересечений. В моменты самопересечений вершины выбираются следующим образом. В первый из отмеченных моментов самопересечения $t_{j_{1}}$ мы можем выбрать вершину из числа тех, которые появились в пути до $t_{j_{1}}$-го момента времени; таких вершин ровно $j_{1}-1$, поскольку траектория случайного блуждания $x(t)$ прошла $j_{1}-1$ шагов направо при $0 \leqslant t<t_{j_{1}}$. В следующий момент самопересечения $t_{j_{2}}$ существует ровно $j_{2}-2$ возможностей для выбора вершины (пока мы рассматриваем простые самопересечения, вершина, выбранная в $t_{j_{1}}$-й момент времени, не может появиться в следующие моменты самопересечений). Аналогично, в последний из отмеченных моментов самопересечения $t_{j_{M}}$ существует ровно $j_{M}-M$ возможностей для выбора очередной вершины. В случае пути с самопересечениями выбор вершин в неотмеченные моменты времени (выбор «траектории возвращения назад») может быть неоднозначен. При первом «возвращении назад» из вершины простого самопересечения можно идти по одному из трех ребер:

а) по ребру, по которому мы пришли в эту вершину в первый раз;

б) по ребру, по которому мы ушли из этой вершины;

в) по ребру, по которому мы пришли в эту вершину во второй раз.

Как мы покажем ниже, для типичных путей реализуется только третья возможность, поскольку ребра а) и б) к моменту самопересечения будут уже пройдены повторно. Обозначим через $Z_{1}(M)$ сумму по путям с $M$ простыми самопересечениями, для которых при «возвращении назад» всегда выбираем ребро, по которому пришли в вершину самопересечения в последний раз. Если каждое ребро пути $\mathscr{P}$ пройдено ровно два раза, то

$$
\mathbf{E}\left(\prod_{l=0}^{2 s_{n}-1} \xi_{i_{l} i_{l+1}}\right)=\left(\frac{1}{4}\right)^{s_{n}} .
$$

В общем случае некоторые ребра могут проходиться четыре раза. Обозначим число таких ребер через $m$. Тогда 


$$
\mathbf{E}\left(\prod_{l=0}^{2 s_{n}-1} \xi_{l_{l} i_{l+1}}\right) \leqslant\left(\frac{1}{4}\right)^{s_{n}-2 m}\left(\text { const }_{2}\right)^{m} .
$$

Покажем, что основной вклад в $Z_{1}(M)$ вносится путями, при которых каждое ребро проходится два раза. В приложении мы изучаем следующую характеристику пути $\mathscr{P}$ - максимальное количество вершин, в которые можно прийти в отмеченные моменты времени из какой-либо одной вершины. Обозначим это число через $\nu_{n}(\mathscr{P})$. Согласно определению, любая вершина $i$ пути $\mathscr{P}$ является левым концом не более $\nu_{n}(\mathscr{P})$ отмеченных ребер. В приложении доказывается, что для типичных путей величина $\nu_{n}(\mathscr{P})$ не может расти слишком быстро; она растет, например, не быстрее, чем $s_{n}^{\gamma}, 0<\gamma \leqslant 1$. Иными словами, сумма по путям, для которых $\nu_{n}(\mathscr{P})>s_{n}^{\gamma}$, есть $o(1)$ (см. лемму 2). В дальнейшем мы всегда предполагаем, что

$$
\nu_{n}(\mathscr{P})<s_{n}^{1 / 4} .
$$

Пусть $j_{u_{1}}, \ldots, j_{u_{m}}$ - индексы моментов самопересечений, отвечающих ребрам, проходимым четыре раза. Тогда

$$
\begin{aligned}
Z_{1}(M)=\sum_{X=\{x(t)\}} & \sum_{\substack{1 \leqslant j_{1}<\cdots<j_{M} \leqslant s_{n} \\
\times\left(j_{1}-1\right)\left(j_{2}-2\right) \cdots\left(j_{M}-M\right) \frac{1}{n^{s_{n}}}}} \mathbf{E}\left(\prod_{l=0}^{2 s_{n}-1} \xi_{i_{l} i_{l+1}}\right) .
\end{aligned}
$$

Вероятность выбора вершины самопересечения, отвечающей ребру, проходимому четыре раза, имеет порядок $\nu_{n}(\mathscr{P}) / s_{n}$, и среднее число таких вершин есть $O\left(M \nu_{n}(\mathscr{P}) / s_{n}\right)=o(1)$, так как $\nu_{n}(\mathscr{P})<s_{n}^{1 / 4}$ и $M<10 s_{n}^{2} / n \ll s_{n}^{1 / 2}$. Более аккуратно приведенные выше рассуждения проводятся следующим обра3ом:

$$
\begin{aligned}
Z_{1}(M)=\sum_{X} & \sum_{\substack{1 \leqslant j_{1}<\cdots<j_{M} \leqslant s_{n} \\
\times}} n(n-1) \cdots\left(n-s_{n}+M\right) \\
& \times\left(j_{1}-1\right)\left(j_{2}-2\right) \cdots\left(j_{M}-M\right) \frac{1}{n^{s_{n}}}\left(\frac{1}{4}\right)^{s_{n}}+Z_{1}^{\prime}(M),
\end{aligned}
$$

где

$$
\begin{aligned}
Z_{1}^{\prime}(M) \leqslant & \sum_{X} \sum_{1 \leqslant j_{1}<\cdots<j_{M} \leqslant s_{n}} \sum_{m=1}^{M} n(n-1) \cdots\left(n-s_{n}+M\right) \\
& \times \sum_{1 \leqslant u_{1}<\cdots<u_{m} \leqslant M}\left(j_{1}-1\right) \cdots\left(\widehat{j_{u_{1}}-u_{1}}\right) \cdots\left(j_{u_{m}-u_{m}}\right) \cdots\left(j_{M}-M\right) \\
& \times\left(s_{n}^{1 / 4}\right)^{m} \frac{1}{n^{s_{n}}}\left(\frac{1}{4}\right)^{s_{n}-2 m}\left(\text { const }_{2}\right)^{m}
\end{aligned}
$$

(здесь запись $\left(\widehat{j_{u_{1}}-u_{1}}\right) \cdots\left(j_{u_{m}-u_{m}}\right)$ означает отсутствие этих множителей в произведении). Подсумма $Z_{1}^{\prime}(M)$ отвечает путям $\mathscr{P}$ с ребрами, проходимыми четыре раза. Обозначим через $B(M)$ подсумму

$$
\sum_{1 \leqslant j_{1}<\cdots<j_{M} \leqslant s_{n}}\left(j_{1}-1\right) \cdots\left(j_{M}-M\right)
$$


в формуле (4.12). Справедлива следующая оценка:

$$
\begin{aligned}
B(M) & =\sum_{0 \leqslant l_{1} \leqslant \cdots \leqslant l_{M} \leqslant s_{n}-M} l_{1} \cdots l_{M}=\frac{1}{M !}\left(\sum_{0}^{s_{n}-M} l\right)^{M}\left(1+O\left(\frac{s_{n}}{n^{2 / 3}}\right)\right) \\
& =\frac{1}{M !}\left(\frac{\left(s_{n}-M\right)\left(s_{n}-M+1\right)}{2}\right)^{M}\left(1+O\left(\frac{s_{n}}{n^{2 / 3}}\right)\right)=\frac{\left(s_{n}^{2} / 2\right)^{M}}{M !}(1+o(1))
\end{aligned}
$$

равномерно по $0 \leqslant M \leqslant 10 s_{n}^{2} / n$. Из (4.14) получаем, что

$$
\begin{aligned}
Z_{1}(M) & =\frac{\left(2 s_{n}\right) !}{s_{n} !\left(s_{n}+1\right) !} n \prod_{k=1}^{s_{n}-M}\left(1-\frac{k}{n}\right) \frac{B(M)}{n^{M}}\left(\frac{1}{4}\right)^{s_{n}}(1+o(1))+Z_{1}^{\prime}(M) \\
& =\frac{1}{\sqrt{\pi}} \frac{n}{s_{n}^{3 / 2}} \exp \left(-\frac{s_{n}^{2}}{2 n}\right) \frac{\left(s_{n}^{2} / 2 n\right)^{M}}{M !}(1+o(1))+Z_{1}^{\prime}(M)
\end{aligned}
$$

Проводя аналогичные оценки для $Z_{1}^{\prime}(M)$, получаем, что

$$
Z_{1}^{\prime}(M) \leqslant O\left(\frac{M s_{n}^{1 / 4}}{s_{n}}\right) \frac{1}{\sqrt{\pi}} \frac{n}{s_{n}^{3 / 2}} \exp \left(-\frac{s_{n}^{2}}{2 n}\right) \frac{\left(s_{n}^{2} / 2 n\right)^{M}}{M !}
$$

равномерно по $0 \leqslant M \leqslant 10 s_{n}^{2} / n$. Таким образом,

$$
Z_{1}(M)=\frac{1}{\sqrt{\pi}} \frac{n}{s_{n}^{3 / 2}} \exp \left(-\frac{s_{n}^{2}}{2 n}\right) \frac{\left(s_{n}^{2} / 2 n\right)^{M}}{M !}(1+o(1)),
$$

и, суммируя по всем $M$, получаем, что

$$
Z_{1}=\sum_{M=0}^{s_{n}} Z_{1}(M)=\sum_{M=0}^{10 s_{n}^{2} / n} Z_{1}(M)+o(1)=\frac{1}{\sqrt{\pi}} \frac{n}{s_{n}^{3 / 2}}(1+o(1)) .
$$

Вершины самопересечения, для которых существует более одной возможности для «возвращения назад», будем называть незакрытыми. Обозначим их число через $r=r(\mathscr{P})$. Кроме того, обозначим моменты самопересечений, соответствуюшие незакрытым вершинам, через $t_{j_{k_{l}}}, l=1, \ldots, r$. Очевидно, что вершина является незакрытой, только если ребро, по которому мы пришли в эту вершину в первый раз, не было пройдено «назад» (т.е. в неотмеченный момент времени) до момента самопересечения. Поэтому число возможностей для выбора незакрытой вершины самопересечения в момент $t$ не превосходит $x(t)$. Необходимо также учесть, что в общем случае геометрия самопересечений может быть более сложной - кроме простых самопересечений могут появляться тройные, четверные и т.п.

Пусть $\mathscr{P}$ насчитывает $M$ моментов самопересечений, т.е.

$$
M=\sum_{k=2}^{s_{n}}(k-1) n_{k}
$$


Обозначим через $j_{1}, \ldots, j_{n_{2}}, 1 \leqslant j_{1}<\cdots<j_{n_{2}} \leqslant s_{n}$, индексы моментов простых самопересечений, а через $r$ число незакрытых вершин, и пусть $j_{l_{1}}, \ldots, j_{l_{r}}$ - индексы моментов простых самопересечений, отвечающих незакрытым вершинам. Пары индексов отмеченных моментов, отвечающих вершинам класса $N_{3}$, мы обозначим через

$$
\left(j_{1,1}^{(2)}, j_{1,2}^{(2)}\right),\left(j_{2,1}^{(2)}, j_{2,2}^{(2)}\right), \ldots,\left(j_{n_{3}, 1}^{(2)}, j_{n_{3}, 2}^{(2)}\right),
$$

упорядочив их так, что

$$
1 \leqslant j_{1,1}^{(2)}<j_{2,1}^{(2)}<\cdots<j_{n_{3}, 1}^{(2)} \leqslant s_{n}, \quad j_{k, 1}^{(2)}<j_{k, 2}^{(2)}, \quad k=1, \ldots, n_{3} .
$$

Мы предполагаем, что в момент времени $t_{j_{k, 1}^{(2)}}$ мы попадаем в $k$-ю вершину тройного пересечения во второй раз (в отмеченный момент времени), а в $t_{j_{k, 2}^{(2)}}$ - в третий.

Аналогично через

$$
\left(j_{1,1}^{(3)}, j_{1,2}^{(3)}, j_{1,3}^{(3)}\right), \ldots,\left(j_{n_{4}, 1}^{(3)}, j_{n_{4}, 2}^{(3)}, j_{n_{4}, 3}^{(3)}\right)
$$

мы обозначим тройки индексов моментов самопересечений, отвечающих вершинам класса $N_{4}$, причем считаем, что

$$
1 \leqslant j_{1,1}^{(3)}<j_{2,1}^{(3)}<\cdots<j_{n_{4}, 1}^{(3)} \leqslant s_{n}, \quad j_{k, 1}^{(3)}<j_{k, 2}^{(3)}<j_{k, 3}^{(3)}, \quad k=1, \ldots, n_{4},
$$

и т.д. Обозначим через $Z_{2}$ сумму по путям, допускающим только простые самопересечения, но в которых число незакрытых вершин отлично от нуля, а через $Z_{3}$ сумму по путям, допускающим самопересечения, отличные от простых. Ниже показывается, что $Z_{2}$ и $Z_{3}$ малы по сравнению с $Z_{1}$, т. е.

$$
Z_{2}=o\left(n / s_{n}^{3 / 2}\right), \quad Z_{3}=o\left(n / s_{n}^{3 / 2}\right) .
$$

Рассмотрим сначала случай $Z_{2}$. Согласно предыдущим рассуждениям, справедлива такая оценка сверху:

$$
\begin{aligned}
Z_{2} \leqslant \sum_{X} & \sum_{M=1}^{10 s_{n}^{2} / n} \sum_{1 \leqslant j_{1}<\cdots<j_{M} \leqslant s_{n}} \sum_{r=1}^{M} \sum_{1 \leqslant l_{1}<\cdots<l_{r} \leqslant M} n(n-1) \cdots\left(n-s_{n}+M\right) \\
& \times\left(j_{1}-1\right) \cdots\left(\widehat{j_{l_{1}}-l_{1}}\right) \cdots\left(\widehat{j_{l_{r}}-l_{r}}\right) \cdots\left(j_{M}-M\right) \\
& \times x\left(t_{j_{l_{1}}}\right) \cdots x\left(t_{j_{l_{r}}}\right) \cdot \frac{1}{n^{s_{n}}} \cdot \mathbf{E}\left(\prod_{k=0}^{2 s_{n}-1} \xi_{i_{k} i_{k+1}}\right) \cdot W_{n}+o(1) .
\end{aligned}
$$

Последний множитель в формуле (4.21), обозначенный через $W_{n}$, задает число способов продолжения траектории в неотмеченные моменты времени при условии, что в отмеченные моменты времени вершины уже выбраны.

Если все самопересечения пути $\mathscr{P}$ простые и существует ровно $r$ незакрытых вершин, то $W_{n} \leqslant 3^{r}$. Двойная сумма

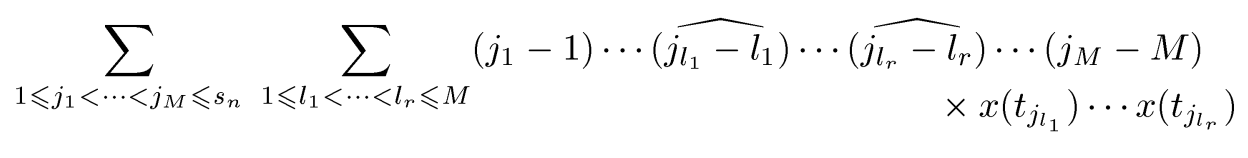


оценивается сверху величиной

$$
\begin{gathered}
\frac{1}{M !}\left(\sum_{j=1}^{s_{n}}(j-1)\right)^{M-r}\left(\sum_{l=1}^{s_{n}} 1\right)^{r} \frac{1}{r !}\left(\sum_{k=1}^{M} 1 \cdot \max _{0 \leqslant t \leqslant 2 s_{n}} x(t)\right)^{r} \\
\leqslant \frac{1}{M !}\left(\frac{s_{n}^{2}}{2}\right)^{M} \frac{1}{r !}\left(\frac{2 M}{\sqrt{s_{n}}} \max _{0 \leqslant t \leqslant 2 s_{n}} \frac{x(t)}{\sqrt{s_{n}}}\right)^{r} .
\end{gathered}
$$

Таким образом, подсумма $Z_{2}$ по путям, в которых каждое ребро проходится ровно два раза, не превосходит

$$
\begin{aligned}
& n \frac{\left(2 s_{n}\right) !}{s_{n} !\left(s_{n}+1\right) !}\left(\frac{1}{4}\right)^{s_{n}} e^{-s_{n}^{2} / 2 n}(1+o(1)) \\
& \quad \times\left(\sum_{M=0}^{\infty} \frac{\left(s_{n}^{2} / 2 n\right)^{M}}{M !}\right) \frac{\sum_{X} \sum_{r=1}^{\infty} \frac{1}{r !}\left(\frac{60 s_{n}^{3 / 2}}{n} \max \frac{x(t)}{\sqrt{s_{n}}}\right)^{r}}{\sum_{X} 1}+o(1) \\
& \quad \leqslant \frac{1}{\sqrt{\pi}} \frac{n}{s_{n}^{3 / 2}}(1+o(1))\left(\mathbf{E}_{X}\left(\exp \left(\frac{60 s_{n}^{3 / 2}}{n} \max _{0 \leqslant t \leqslant 2 s_{n}} \frac{x(t)}{\sqrt{s_{n}}}\right)-1\right)\right) .
\end{aligned}
$$

Последний множитель в (4.22) стремится к нулю при $n \rightarrow \infty$. В самом деле, распределение нормированной траектории случайного блуждания $x_{n}(t)=$ $\left(x\left(\left[2 s_{n} t\right]\right)+\left(2 s_{n} t-\left[2 s_{n} t\right]\right)\left(x\left(\left[2 s_{n} t\right]+1\right)-x\left(\left[2 s_{n} t\right]\right)\right)\right)\left(2 s_{n}\right)^{-1 / 2}, 0 \leqslant t \leqslant 1$, сходится к условному распределению бесселевского процесса на положительной полуоси при условии, что $b(1)=0$. Следовательно, при любом сколь угодно малом $\varepsilon>0$

$$
\mathbf{E}_{X}\left(\exp \left(\varepsilon \max _{0 \leqslant t \leqslant 2 s_{n}} \frac{x(t)}{\sqrt{2 s_{n}}}\right)\right) \underset{n \rightarrow \infty}{\longrightarrow} \mathbf{E}\left(\exp \left(\varepsilon \max _{0 \leqslant t \leqslant 1} b(t)\right) \mid b(1)=0\right)
$$

и, так как коэффициент $60 s_{n}^{3 / 2} / n$ в формуле (2.22) стремится к нулю при $n \rightarrow \infty$, то

$$
\begin{aligned}
0 & \leqslant \mathbf{E}_{X}\left(\exp \left(\frac{60 s_{n}^{3 / 2}}{n} \max _{0 \leqslant t \leqslant 2 s_{n}} \frac{x(t)}{\sqrt{s_{n}}}\right)-1\right) \leqslant \mathbf{E}_{X}\left(\exp \left(\varepsilon \max _{0 \leqslant t \leqslant 2 s_{n}} \frac{x(t)}{\sqrt{s_{n}}}\right)-1\right) \\
& \leqslant\left(\mathbf{E}\left(\exp \left(\varepsilon \max _{0 \leqslant t \leqslant 1} b(t) \mid b(1)=0\right)\right)-1\right)(1+\varepsilon) .
\end{aligned}
$$

Выбирая $\varepsilon$ достаточно малым, мы можем сделать правую часть в (4.23) сколь угодно близкой к нулю. Таким образом,

$$
Z_{2}=o\left(Z_{1}\right)+Z_{2}^{\prime}
$$

где $Z_{2}^{\prime}$ есть подсумма в $Z_{2}$ по путям, при которых хотя бы одно ребро проходится четыре раза. Сумма $Z_{2}^{\prime}$ анализируется аналогично $Z_{1}^{\prime}$, и в результате получаем, что $Z_{2}^{\prime}=o\left(Z_{1}\right)$, а это вместе с (4.24) влечет за собой равенство $Z_{2}=o\left(Z_{1}\right)$.

Рассмотрим теперь сумму $Z_{3}$ по путям, которые допускают самопересечения, отличные от простых. Используя введенные выше обозначения, мы 
можем написать следующую оценку сверху для $Z_{3}$ :

$$
\begin{aligned}
& Z_{3} \leqslant \sum_{X=\{x(t)\}} \sum_{M=1}^{10 s_{n}^{2} / n} \sum_{n_{2}+2 n_{3}+\cdots+M n_{M+1}=M} \sum_{r=0}^{n_{2}} \sum_{1 \leqslant j_{1}<\cdots<j_{n_{2}} \leqslant s_{n}} \sum_{1 \leqslant l_{1}<\cdots<l_{r} \leqslant n_{2}} \\
& \sum \sum \\
& \left(j_{1,1}^{(2)}, j_{1,2}^{(2)}\right), \ldots,\left(j_{n_{3}, 1}^{(2)}, j_{n_{3}, 2}^{(2)}\right), \quad\left(j_{1,1}^{(3)}, j_{1,2}^{(3)}, j_{1,3}^{(3)}\right), \ldots,\left(j_{n_{4}, 1}^{(3)}, j_{n_{4}, 2}^{(3)}, j_{n_{4}, 3}^{(3)}\right), \\
& 1 \leqslant j_{1,1}^{(2)}<j_{2,1}^{(2)}<\cdots<j_{n_{3}, 1}^{(2)} \leqslant s_{n}, \quad 1 \leqslant j_{1,1}^{(3)}<j_{2,1}^{(3)}<\cdots<j_{n_{4}, 1}^{(3)} \leqslant s_{n}, \\
& j_{q_{2}, 1}^{(2)}<j_{q_{2}, 2}^{(2)}, q_{2}=1, \ldots, n_{3} \quad j_{q_{3}, 1}^{(3)}<j_{q_{3}, 2}^{(3)}<j_{q_{3}, 3}^{(3)}, q_{3}=1, \ldots, n_{4} \\
& n(n-1) \cdots\left(n-s_{n}+M\right)\left(j_{1}-1\right) \cdots\left(\widehat{j_{l_{1}}-l_{1}}\right) \cdots\left(\widehat{j_{l_{r}}-l_{r}}\right) \cdots\left(j_{n_{2}}-n_{2}\right) \\
& \times \prod_{k=1}^{r} x\left(t_{j_{l_{k}}}\right) \cdot \prod_{q_{2}=1}^{n_{3}}\left(j_{q_{2}, 1}^{(2)}-1\right) \cdot \prod_{q_{3}=1}^{n_{4}}\left(j_{q_{3}, 1}^{(3)}-1\right) \cdots \\
& \times \frac{1}{n^{s_{n}}} \cdot \mathbf{E}\left(\prod_{u=0}^{2 s_{n}-1} \xi_{i_{u} i_{u+1}}\right) W_{n} .
\end{aligned}
$$

Через $W_{n}$ мы по-прежнему обозначаем число способов, с помощью которых, зная траекторию в отмеченные моменты времени, можно выбрать вершины пути в неотмеченные моменты времени. В [14] была по-существу использована следующая оценка:

$$
W_{n} \leqslant \prod_{k=3}^{M}(2 k)^{k n_{k}} 3^{r} .
$$

Этой оценки было бы достаточно для наших нужд в случае равномерно ограниченных случайных величин $\left\{\xi_{i j}\right\}$. В общем случае, когда моменты случайных величин $\xi_{i j}$ растут сверхэкспоненциально, множитель $\mathbf{E}\left(\prod_{u=0}^{2 s_{n}-1} \xi_{i_{u} i_{u+1}}\right)$ может быть большим за счет ребер, проходимых большое число раз, и требуется несколько более тонкая оценка последних двух множителей в (4.25), доказываемая ниже.

ЛЕмма 1.

$$
\mathbf{E}\left(\prod_{u=0}^{2 s_{n}-1} \xi_{i_{u} i_{u+1}}\right) W_{n} \leqslant\left(\frac{1}{4}\right)^{s_{n}} \prod_{k=3}^{M}\left(\text { const }_{3} \cdot k\right)^{k n_{k}} 3^{r} .
$$

ДокАЗАТЕЛЬСТВо ЛЕмМЫ. В [14] использовалась оценка (4.26) для $W_{n}$ и оценка

$$
\mathbf{E}\left(\prod_{u=0}^{2 s_{n}-1} \xi_{i_{u} i_{u+1}}\right) \leqslant\left(\frac{1}{4}\right)^{s_{n}} \prod_{\text {ребра }\{i, j\} \text { пути } \mathscr{P}}(4 \text { const } \cdot l(\{i, j\}))^{l(\{i, j\})},
$$

где через $\{i, j\}$ мы обозначаем ребра пути $\mathscr{P}$, а $2 l(\{i, j\})$ - число раз, которое путь $\mathscr{P}$ проходит ребро $\{i, j\}$. Оценка (4.26) вытекает из того, что для вершины $k$-кратного пересечения при каждом "возвращении назад» для этого существует не более $2 k$ возможностей. Оценка (4.28) есть прямое 
следствие условия (1.1) на рост моментов случайной величины $\left\{\xi_{i j}\right\}$. Смысл леммы 1 заключается в том, что эти два множителя не могут быть одновременно большими. Как только какое-либо ребро проходится больше чем два раза, скажем $2 l>2$, что влечет за собой появление множителя $(4 \text { const } \cdot l)^{l}$ в правой части формулы (4.28), то одновременно мы можем сократить оценку для $W_{n}$ в правой части формулы (4.26) в $l$ ! раз, так как «возвращение назад» происходит $l$ раз по одному и тому же ребру. Таким образом,

$$
\begin{aligned}
& \mathbf{E}\left(\prod_{u=0}^{2 s_{n}-1} \xi_{i_{u} i_{u+1}}\right) W_{n} \\
& \quad \leqslant\left(\frac{1}{4}\right)^{s_{n}} \prod_{\{i, j\}}^{\prime}(4 \text { const } \cdot l(\{i, j\}))^{l(\{i, j\})} \frac{1}{l(\{i, j\}) !} \prod_{k=2}^{M}(2 k)^{k n_{k}} 3^{r} \\
& \quad \leqslant\left(\frac{1}{4}\right)^{s_{n}} \prod_{\{i, j\}}^{\prime}\left(\operatorname{const}_{1}\right)^{l(\{i, j\})} \prod_{k=3}^{M}(2 k)^{k n_{k}} 3^{r} \\
& \leqslant\left(\frac{1}{4}\right)^{s_{n}}\left(\operatorname{const}_{1}\right)^{\sum_{k=3}^{M} k n_{k}} \prod_{k=3}^{M}(2 k)^{k n_{k}} 3^{r} .
\end{aligned}
$$

Это и есть оценка из леммы 1. В оценке (4.29) произведение $\prod_{\{i, j\}}^{\prime}$ рассматривалось только по ребрам $\{i . j\}$, таким, что $l(\{i, j\}) \geqslant 4$.

Дальнейшие оценки для $Z_{3}$ проводятся аналогично случаю $Z_{2}$ и $Z_{1}$. А именно, рассмотрим подсумму в (4.25) вида

$$
\begin{aligned}
& \sum_{1 \leqslant j_{1}<\cdots<j_{n_{2}} \leqslant s_{n}} \sum_{1 \leqslant l_{1}<\cdots<l_{r} \leqslant n_{2}} \sum_{\left(j_{1,1}^{(2)}, j_{1,2}^{(2)}\right), \ldots,\left(j_{n_{3}, 1}^{(2)}, j_{n_{3}, 2}^{(2)}\right),} \sum_{\left(j_{1,1}^{(3)}, j_{1,2}^{(3)}, j_{1,3}^{(3)}\right), \ldots,} \\
& 1 \leqslant j_{1,1}^{(2)}<\cdots<j_{n_{3}, 1}^{(2)} \leqslant s_{n}, \quad\left(j_{n_{4}, 1}^{(3)}, j_{n_{4}, 2}^{(3)}, j_{n_{4}, 3}^{(3)}\right), \\
& j_{q_{2}, 1}^{(2)}<j_{q_{2}, 2}^{(2)}, q_{2}=1, \ldots, n_{3} \quad 1 \leqslant j_{1,1}^{(3)}<\cdots<j_{n_{4}, 1}^{(3)} \leqslant s_{n} \\
& \left(j_{1}-1\right) \ldots\left(\widehat{j_{l_{1}}-l_{1}}\right) \cdots\left(\widehat{j_{l_{r}}-l_{r}}\right) \cdots\left(j_{n_{2}}-n_{2}\right) \\
& \times \prod_{k=1}^{r} x\left(t_{j_{l_{k}}}\right) \cdot \prod_{q_{2}=1}^{n_{3}}\left(j_{q_{2}, 1}^{(2)}-1\right) \cdot \prod_{q_{3}=1}^{n_{4}}\left(j_{q_{3}, 1}^{(3)}-1\right) \cdots \prod_{q_{M}=1}^{n_{M+1}}\left(j_{g_{M}, 1}^{(M)}-1\right) .
\end{aligned}
$$

Подсумма (4.30) оценивается сверху величиной

$$
\begin{gathered}
\frac{1}{n_{2} !}\left(\sum_{j_{1}=1}^{s_{n}}\left(j_{1}-1\right)\right)^{n_{2}-r} \frac{1}{r !}\left(\sum_{j_{2}=1}^{s_{n}} 1\right)^{r}\left(\sum_{l=1}^{M} \max _{0 \leqslant t \leqslant 2 s_{n}} x(t) \cdot 1\right)^{r} \\
\quad \times \frac{1}{n_{3} !}\left(\frac{1}{2 !} \sum_{j_{3}=1}^{s_{n}}\left(j_{3}-1\right) \cdot \sum_{j_{4}=1}^{s_{n}} 1\right)^{n_{3}} \frac{1}{n_{4} !}\left(\frac{1}{3 !} \sum_{j_{5}=1}^{s_{n}}\left(j_{5}-1\right) \cdot \sum_{j_{6}=1}^{s_{n}} 1 \cdot \sum_{j_{7}=1}^{s_{n}} 1\right)^{n_{4}} \ldots \\
\leqslant \prod_{k=2}^{s_{n}} \frac{1}{n_{k} !}\left(\frac{s_{n}^{k}}{2} \frac{1}{(k-1) !}\right)^{n_{k}} \frac{1}{r !}\left(\frac{2 M}{\sqrt{s_{n}}} \max \frac{x(t)}{\sqrt{s_{n}}}\right)^{r} .
\end{gathered}
$$


Из (4.31) и леммы 1 следует, что

$$
\begin{aligned}
Z_{3} \leqslant n & \frac{\left(2 s_{n}\right) !}{s_{n} !\left(s_{n}+1\right) !}\left(\frac{1}{4}\right)^{s_{n}} \sum_{M=1}^{10 s_{n}^{2} / n} \sum_{n_{2}+2 n_{3}+\cdots+M n_{M+1}=M} \prod_{i=1}^{s_{n}-M}\left(1-\frac{i}{n}\right) \\
& \times \frac{1}{n_{2} !}\left(\frac{s_{n}^{2}}{2 n}\right)^{n_{2}} \cdot \prod_{k=3}^{s_{n}+1} \frac{1}{n_{k} !}\left(\frac{s_{n}^{k}}{2 n^{k-1}} \frac{\left(\mathrm{const}_{2} \cdot k\right)^{k}}{(k-1) !}\right)^{n_{k}} \\
+ & \frac{\left(2 s_{n}\right) !}{s_{n} !\left(s_{n}+1\right) !}\left(\frac{1}{4}\right)^{s_{n}} \sum_{M=1}^{10 s_{n}^{2} / n} \sum_{n_{2}+2 n_{3}+\cdots+M n_{M+1}=M} \prod_{i=1}^{s_{n}-M}\left(1-\frac{i}{n}\right) \\
\times & \frac{1}{n_{2} !}\left(\frac{s_{n}^{2}}{2 n}\right)^{n_{2}} \cdot \prod_{k=3}^{s_{n}} \frac{1}{n_{k} !}\left(\frac{s_{n}^{k}}{2 n^{k-1}} \frac{\mathrm{const}_{2} \cdot k^{k}}{(k-1) !}\right)^{n_{k}} \\
\times & \mathbf{E}_{X}\left(\exp \left(\frac{60 s_{n}^{3 / 2}}{n} \max _{0 \leqslant t \leqslant 2 s_{n}} \frac{x(t)}{\sqrt{s_{n}}}\right)-1\right)+o(1) \\
\leqslant \frac{n}{\sqrt{\pi} s_{n}^{3 / 2}} & \left\{\left(\exp \left(\sum_{k=3}^{s_{n}+1} \frac{s_{n}^{k}}{2 n^{k-1}} \operatorname{const}_{5}^{k}\right)-1\right)\right. \\
& \left.+\mathbf{E}_{X}\left(\exp \left(\frac{60 s_{n}^{3 / 2}}{n} \max _{0 \leqslant t \leqslant 2 s_{n}} \frac{x(t)}{\sqrt{s_{n}}}\right)-1\right)\right\}=o\left(\frac{n}{s_{n}^{3 / 2}}\right) .
\end{aligned}
$$

Таким образом, формулы (4.20) доказаны, что вместе с (4.17) влечет за собой оценку для главного порядка математического ожидания:

$$
\mathbf{E}\left(\operatorname{Tr} A_{n}^{2 s_{n}}\right)=\frac{n}{\sqrt{\pi s_{n}^{3}}}(1+o(1)) \quad \text { при } s_{n} \ll n^{2 / 3} .
$$

Попутно нами получена следующая оценка для числа четных путей длины $2 s_{n}$ :

ПрЕДЛОЖЕНИЕ 2. Пусть $1 \ll s_{n} \ll n^{2 / 3}$. Тогда число четных путей длинь $2 s_{n}$ есть $n^{s_{n}+1} 4^{s_{n}} \pi^{-1 / 2} s_{n}^{-3 / 2}(1+o(1))$.

\section{§5. Оценка дисперсии и старших моментов случайной величины $\operatorname{Tr} A_{n}^{p_{n}}$}

Случай дисперсии и старших моментов случайной величины $\operatorname{Tr} A^{p_{n}}$ pacсматривается в основном так же, как в [14]. Ниже для удобства читателей мы приводим основные идеи доказательства.

ПредлОЖенИЕ 3. Пусть $1 \ll p_{n} \ll n^{2 / 3}$. Тогда $\operatorname{Var}\left(\operatorname{Tr} A_{n}^{p_{n}}\right) \leqslant$ const $_{6} \partial_{\Omega} \Omega$ всех $n$ u $\operatorname{Var}\left(\operatorname{Tr} A_{n}^{p_{n}}\right) \rightarrow 1 / \pi$ npu $n \rightarrow \infty, p_{n} \rightarrow \infty, p_{n} n^{-2 / 3} \rightarrow 0$. 
Формула для дисперсии случайной величины $\operatorname{Tr} A^{p_{n}}$ имеет вид

$$
\begin{aligned}
\operatorname{Var}\left(\operatorname{Tr} A_{n}^{p_{n}}\right)= & \mathbf{E}\left(\operatorname{Tr} A_{n}^{p_{n}}\right)^{2}-\left(\mathbf{E}\left(\operatorname{Tr} A_{n}^{p_{n}}\right)\right)^{2} \\
= & \sum_{i_{0}, i_{1}, \ldots, i_{p_{n}-1}=1}^{n} \sum_{j_{0}, j_{1}, \ldots, j_{p_{n}-1}=1}^{n} \frac{1}{n^{p_{n}}} \cdot\left(\mathbf{E}\left(\prod_{l=1}^{p_{n}} \xi_{i_{l-1} i_{l}} \cdot \prod_{m=1}^{p_{n}} \xi_{j_{m-1} j_{m}}\right)\right. \\
& \left.-\mathbf{E}\left(\prod_{l=1}^{p_{n}} \xi_{i_{l-1} i_{l}}\right) \cdot \mathbf{E}\left(\prod_{m=1}^{p_{n}} \xi_{j_{m-1} j_{m}}\right)\right),
\end{aligned}
$$

где мы считаем, что $i_{p_{n}}=i_{0}, j_{p_{n}}=j_{0}$.

Вследствие независимости симметрично распределенных случайных величин $\xi_{i j}, i \leqslant j$, большинство слагаемых в (5.1) обращается в нуль. Слагаемое, отвечающее паре замкнутых путей длины $p_{n}$

$$
\mathscr{P}=\left\{i_{0} \rightarrow i_{1} \rightarrow \cdots \rightarrow i_{p_{n}-1} \rightarrow i_{0}\right\}, \quad \mathscr{P}^{\prime}=\left\{j_{0} \rightarrow j_{1} \rightarrow \cdots \rightarrow j_{p_{n}-1} \rightarrow j_{0}\right\}
$$

не равно нулю тогда и только тогда, когда

а) пути $\mathscr{P}$ и $\mathscr{P}^{\prime}$ имеют хотя бы одно общее (неориентированное) ребро,

б) каждое ребро появляется в объединении $\mathscr{P} \cup \mathscr{P}^{\prime}$ четное число раз (здесь под объединением $\mathscr{P} \cup \mathscr{P}^{\prime}$ мы понимаем объединение множеств ребер путей $\mathscr{P}$ и $\mathscr{P} \prime$, рассматриваемых с кратностями прохождения).

ОПРЕдЕлЕниЕ 5. Каждая упорядоченная пара путей, удовлетворяющая условиям а) и б), называется коррелированной. Коррелированная пара называется просто коррелированной, если каждое ребро появляется в объединении путей $\mathscr{P}$ и $\mathscr{P}^{\prime}$ ровно два раза.

Вычисление $\operatorname{Var}\left(\operatorname{Tr} A^{p_{n}}\right)$ по существу сводится к подсчету числа коррелированных пар путей, рассматриваемых с соответствующим статистическим весом. В частности, из наших рассуждений будет следовать

ПРЕДЛОЖЕНИЕ 4. Пусть $1 \ll p_{n} \ll n^{2 / 3}$. Тогда в главном порлдке число коррелированных пар путей равно числу просто коррелированных пар путей и равно $\pi^{-1} n^{p_{n}} 2^{2 p_{n}}(1+o(1))$.

Техника, развитая в $\S 4$, позволяет ответить на вопрос о главном порядке числа четных путей длины $2 s_{n}$. В самом деле, если случайные величины $\xi_{i j}$, $i \leqslant j$, принимают значения $\pm 1 / 2$ с равной вероятностью, то

$$
\mathbf{E}\left(\operatorname{Tr} A^{2 s_{n}}\right)=4^{s_{n}} n^{s_{n}} \cdot\left(\text { число четных путей длины } 2 s_{n}\right)
$$

и предложение 2 следует из (4.33).

Ниже каждой коррелированной паре путей длины $p_{n}$ ставится в соответствие некоторый четный путь длины $2 p_{n}-2$. Построенное соответствие не будет взаимно однозначным, и каждый путь длины $2 p_{n}-2$ будет иметь, вообще говоря, несколько прообразов. В результате подсчет числа коррелированных пар путей сводится к подсчету числа четных путей, взятых с кратностями, равными числу прообразов. Способ построения соответствия 
проиллюстрирован на рис. 1 и 2.

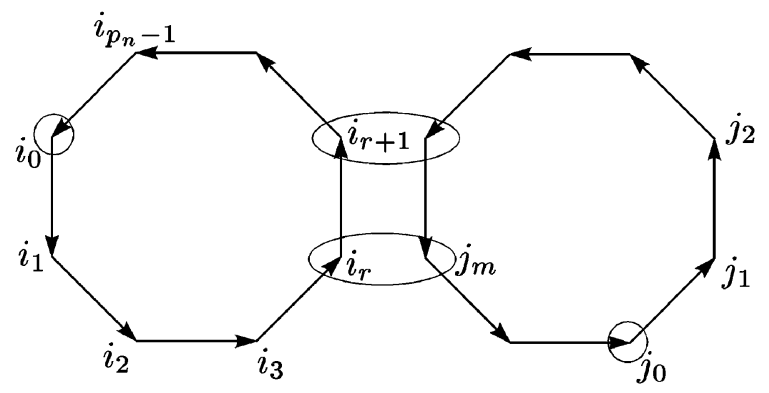

Рис. 1

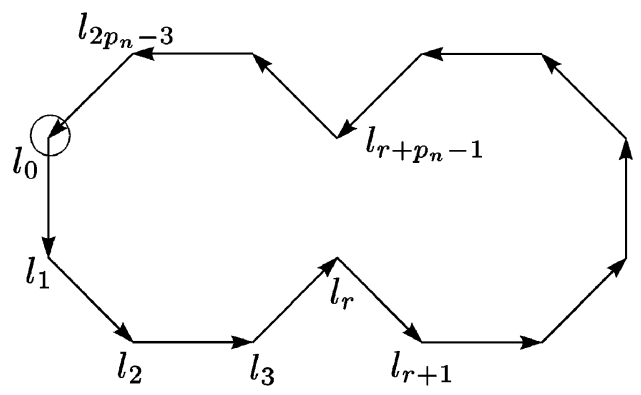

Рис. 2

ОПРЕДЕлЕНИЕ 6. Первым общим ребром упорядоченной коррелированной пары называется первое из ребер пути $\mathscr{P}$, которое также принадлежит $\mathscr{P}^{\prime}$.

Новый путь длины $2 p_{n}-2$ будет состоять из ребер путей $\mathscr{P}$ и $\mathscr{P}$ '. Обозначим его $\mathscr{P} \vee \mathscr{P}^{\prime}$. Путь $\mathscr{P} \vee \mathscr{P}^{\prime}$ строится следующим образом: сначала идем вдоль $\mathscr{P}$ до левого конца первого общего ребра путей $\mathscr{P}$ и $\mathscr{P} \prime$, после чего перескакиваем на $\mathscr{P}^{\prime}$ и делаем $p_{n}-1$ шагов по $\mathscr{P}{ }^{\prime}$ (вдоль $\mathscr{P}{ }^{\prime}$, если направления путей $\mathscr{P}$ и $\mathscr{P}^{\prime}$ в общем ребре различны, или в направлении, противоположном $\mathscr{P}^{\prime}$, если направления путей $\mathscr{P}$ и $\mathscr{P}^{\prime}$ в общем ребре совпадают). Сделав $p_{n}-1$ шагов по $\mathscr{P}$, опять приходим в общее ребро путей $\mathscr{P}$ и $\mathscr{P}^{\prime}$, после чего переходим на $\mathscr{P}$ и заканчиваем путь $\mathscr{P} \vee \mathscr{P}^{\prime}$ вдоль первого пути. Построенный таким образом путь

$$
\begin{aligned}
& \mathscr{P} \vee \mathscr{P}^{\prime}=\left\{l_{0}=i_{0} \rightarrow \cdots \rightarrow l_{r}=i_{r}=j_{m} \rightarrow l_{r+p_{n}-1}=i_{r+1}=j \rightarrow\right. \\
& \left.\cdots \rightarrow l_{2 p_{n}-3} \rightarrow l_{0}=i_{0}\right\}
\end{aligned}
$$

где $\left\{i_{r}, i_{r+1}\right\}$ - общее ребро путей $\mathscr{P}$ и $\mathscr{P}^{\prime}$, является четным вследствие коррелированности пары $\left(\mathscr{P}, \mathscr{P}^{\prime}\right)$.

Оценим, сколькими способами можно получить путь длины $2 p_{n}-2$ из различных коррелированных пар. Во-первых, необходимо выбрать некоторую вершину $l_{r}$ в первой половине пути, $0 \leqslant r \leqslant p_{n}-1$, и соединить ее с вершиной $l_{r+p_{n}-1}$. Выбор производится таким образом, чтобы ребро $\left\{l_{r}, l_{r+p_{n}-1}\right\}$ являлось первым общим ребром путей $\mathscr{P}$ и $\mathscr{P}^{\prime}$. Кроме этого, необходимо 
выбрать начало второго пути $\mathscr{P}^{\prime}$, что в типичной ситуации может быть сделано $p_{n}$ способами, и направление движения вдоль Я尹', что может быть сделано двумя способами. Условие того, что $\left\{l_{r}, l_{r+p_{n}-1}\right\}$ является первым общим ребром путей $\mathscr{P}$ и $\mathscr{P}^{\prime}$, удобнее переформулировать в терминах случайного блуждания

$$
X=\left\{x(t) \geqslant 0,0 \leqslant t \leqslant 2 p_{n}-2, x(0)=x\left(2 p_{n}-2\right)=0\right\}
$$

на положительной полупрямой. Напомним, что $x(t)-x(t-1)=1$, если в течение первых $t$ шагов пути ребро $\left\{l_{t-1}, l_{t}\right\}$ встретилось нечетное число раз, и $x(t)-x(t-1)=-1$ в противном случае. Тогда необходимое условие для того, чтобы ребро $\left\{l_{r}, l_{r+p_{n}-1}\right\}$ было первым общим ребром пары $\left(\mathscr{P}, \mathscr{P}^{\prime}\right)$, заключается в том, чтобы при $r \leqslant t \leqslant r+p_{n}-1$ (половина времени всего блуждания) траектория $x(t)$ не опускалась ниже уровня $x(r)$. Для типичных путей (т. е. путей с простыми самопересечениями и без незакрытых вершин) это условие является также и достаточным. Обозначим через $K_{n}(x(\cdot))$ число моментов времени $r_{j}, 0 \leqslant r_{j} \leqslant p_{n}-1, j=1, \ldots, K_{n}$, таких, что $x(t) \geqslant x\left(r_{j}\right)$ при $r_{j} \leqslant t \leqslant r_{j}+p_{n}+1 . \mathrm{B}[14$, лемма 1] было доказано, что

$$
\mathbf{E}_{X} K_{n}=2 \sqrt{\frac{p_{n}}{\pi}}(1+o(1))
$$

В $\S 4$ было показано, что равномерное распределение на пространстве замкнутых четных путей длины $2 p_{n}-2$ индуцирует распределение на пространстве траекторий случайного блуждания, которое стремится к равномерному при $n \rightarrow \infty$. Следуя рассуждениям $\S 4$, нетрудно показать, что

(число коррелированных пар длины $p_{n}$ )

$$
=n^{p_{n}} \frac{\left(2 p_{n}-2\right) !}{\left(p_{n}-1\right) ! p_{n} !} \mathbf{E}_{X}\left(K_{n}(x(\cdot))\right) \cdot 2 p_{n} \cdot(1+o(1)) .
$$

Утверждение 4 следует из (5.2) и (5.3). При подсчете $\operatorname{Var}\left(\operatorname{Tr} A_{n}^{p_{n}}\right)$ необходимо также учесть статистический вес

$$
\frac{1}{n^{p_{n}}}\left(\mathbf{E}\left(\prod_{l=1}^{p_{n}} \xi_{i_{l-1} i_{l}} \cdot \prod_{m=1}^{p_{n}} \xi_{j_{m-1} j_{m}}\right)-\mathbf{E}\left(\prod_{l=1}^{p_{n}} \xi_{i_{l-1} i_{l}}\right) \cdot \mathbf{E}\left(\prod_{m=1}^{p_{n}} \xi_{j_{m-1} j_{m}}\right)\right) .
$$

Множитель (5.4) равен $2^{-2 p_{n}}$, если выполнены следующие два условия

а) путь $\mathscr{P} \vee \mathscr{P}^{\prime}$ содержит каждое ребро ровно два раза,

б) путь $\mathscr{P} \vee \mathscr{P}^{\prime}$ не содержит неупорядоченное ребро $\left\{l_{r}, l_{r+p_{n}-1}\right\}$.

Доказательство того, что пути, удовлетворяющие условиям а) и б), являются типичными и вносят основной вклад в $\operatorname{Var}\left(\operatorname{Tr} A^{p_{n}}\right)$, по существу повторяет рассуждения $\S 4$. Доказательство предложения 3 закончено.

Случай старших моментов также рассматривается аналогично тому, как это делалось в [14]. Для доказательства теоремы 2 необходимо показать, что

$$
\begin{aligned}
\mathbf{E}\left(\operatorname{Tr} A_{n}^{p_{n}}-\mathbf{E}\left(\operatorname{Tr} A_{n}^{p_{n}}\right)\right)^{2 k} & =\frac{(2 k-1) ! !}{\pi^{k}}+o(1), \\
\mathbf{E}\left(\operatorname{Tr} A_{n}^{p_{n}}-\mathbf{E}\left(\operatorname{Tr} A_{n}^{p_{n}}\right)\right)^{2 k+1} & =o(1) .
\end{aligned}
$$


Имеет место следующее равенство, аналогичное формуле (5.1):

$$
\begin{aligned}
& \mathbf{E}\left(\operatorname{Tr} A_{n}^{p_{n}}-\mathbf{E}\left(\operatorname{Tr} A_{n}^{p_{n}}\right)\right)^{L} \\
& =\frac{1}{n^{p_{n} L / 2}} \mathbf{E} \prod_{m=1}^{L}\left(\sum_{i_{0}^{(m)}, i_{1}^{(m)}, \ldots, i_{p_{n}-1}^{(m)}=1}^{L}\left(\prod_{r=1}^{p_{n}} \xi_{i_{r-1}^{(m)} i_{r}^{(m)}}-\mathbf{E} \prod_{r=1}^{p_{n}} \xi_{i_{r-1}^{(m)} i_{r}^{(m)}}\right)\right) .
\end{aligned}
$$

Рассмотрим произвольный набор $L$ замкнутых путей длины $p_{n}$

$$
\mathscr{P}_{m}=\left\{i_{0}^{(m)} \rightarrow i_{1}^{(m)} \rightarrow \cdots \rightarrow i_{p_{n}}^{(m)}=i_{0}^{(m)}\right\}, \quad m=1, \ldots, L .
$$

ОПРЕДЕЛЕНИЕ 7. Будем называть пути $\mathscr{P}_{m^{\prime}}, \mathscr{P}_{m^{\prime \prime}}$ пересекаюшимися по ребру, если $\mathscr{P}_{m^{\prime}}, \mathscr{P}_{m^{\prime \prime}}$ имеют обшее (неориентированное) ребро.

ОПределЕниЕ 8 . Подмножество путей $\mathscr{P}_{m_{j_{1}}}, \mathscr{P}_{m_{j_{2}}}, \ldots, \mathscr{P}_{m_{j_{k}}}$ называется кластером пересекающихся путей, если

а) для любой пары $\mathscr{P}_{m_{i}}, \mathscr{P}_{m_{j}}$ существует цепочка путей из этого подмножества, которая начинается с $\mathscr{P}_{m_{i}}$, кончается $\mathscr{P}_{m_{j}}$ и такова, что любые два соседних пути цепочки пересекаются по ребру,

б) при добавлении любого нового пути в это подмножество свойство а) нарушается.

Из определения следует, что множества ребер, относящихся к различным кластерам, не пересекаются. Поэтому и вследствие независимости случайных величин $\left\{\xi_{i j}\right\}_{l \leqslant j}$ математическое ожидание в (5.7) представляется в виде произведения математических ожиданий сомножителей, отвечающих различным кластерам. Формулы (5.5), (5.6) следуют из того факта, что основной вклад в (5.7) отвечает ситуации, когда все кластеры состоят ровно из двух путей. (Очевидно, что если хотя бы один кластер состоит ровно из одного пути, то математическое ожидание этого слагаемого равно нулю).

ЛЕMMA 2.

$$
\begin{array}{r}
\mathbf{E} \frac{1}{n^{p_{n} l / 2}} \prod_{m=1}^{l}\left(\sum_{i_{0}^{(m)}, \ldots, i_{p_{n}-1}^{(m)}=1}^{n}\left(\prod_{r=1}^{p_{n}} \xi_{i_{r-1}^{(m)} i_{r}^{(m)}}-\mathbf{E}\left(\prod_{r=1}^{p_{n}} \xi_{i_{r-1}^{(m)} i_{r}^{(m)}}\right)\right)\right) \\
= \begin{cases}1 / \pi+o(1), & \text { если } l=2, \\
o(1), & \text { если } l>2,\end{cases}
\end{array}
$$

где произведение $\prod^{*}$ в (5.8) рассматривается по путям, образуюиим клаcmep.

Доказательство леммы 2 приведено в [14]. Отметим, что случай $l=2$ есть в точности предложение 3 данной статьи. Случай $l>2$ рассматривается аналогичным образом. Каждому кластеру из $l$ путей ставится в соответствие четный путь длины $l_{p_{n}}-q$, где $l \leqslant q \leqslant 2 l$. Число прообразов при таком соответствии ограничено сверху величиной $K_{n}^{l-1}$, где $K_{n}$ - число моментов времени $t_{i}, i=1, \ldots, K_{n}$, таких, что $t_{i} \leqslant(l-1) p_{n}-q$ и $x(t) \geqslant x\left(t_{i}\right)$ при $t_{i} \leqslant t \leqslant t_{i}+p_{n}-1$. Аналогом формулы (5.2) служит неравенство

$$
\mathbf{E}_{X} K_{n}^{l-1} \leqslant \operatorname{const}_{l} \cdot p_{n}^{(l-1) / 2} .
$$




\section{Приложение}

В этом приложении доказывается, что сумма по путям, в которых какоенибудь ребро проходится четыре или более раз, мала по сравнению со всей суммой (4.1).

Пусть $\mathscr{P}=\left\{i_{0} \rightarrow i_{1} \rightarrow \cdots \rightarrow i_{2 s_{n}}=i_{0}\right\}$ и $\nu_{n}(\mathscr{P})$ - максимальное число вершин, в которые можно попасть в отмеченные моменты времени из какой-либо одной вершины. Согласно определению, любая вершина $i$ пути $\mathscr{P}$ является левым концом не более $\nu_{n}(\mathscr{P})$ отмеченных ребер. Ниже мы показываем, что для типичных путей величина $\nu_{n}(\mathscr{P})$ растет медленнее, чем любая степень величины $s_{n}$.

Лемма 3. Для любого $\gamma, 0<\gamma<1$, подсумма в (4.1) по путям Я尹Р, таким, ито $\nu_{n}(\mathscr{P})>s_{n}^{\gamma}, 0<\gamma<1$, есть о(1), и, таким образом, пренебрежимо мала по сравнению со всей суммой.

Для того чтобы понять, почему $\nu_{n}(\mathscr{P})$ не может быть большой для типичных путей, рассмотрим путь с простыми самопересечениями и без незакрытых вершин. Тогда для того, чтобы какая-нибудь вершина являлась левым концом $\nu_{n}$ отмеченных ребер, для соответствующего случайного блуждания на положительной полуоси $x(t), 0 \leqslant t \leqslant 2 s_{n}$, должен существовать временной интервал $\left[t_{1}, t_{2}\right]$, в течение которого траектория $x(t) \nu_{n} / 2$ раз снижается до уровня $x\left(t_{1}\right)$, но ни разу не пересекает его. В самом деле, для того чтобы сделать каждый новый шаг из вершины $i_{t_{1}}$, мы должны сначала вернуться к ней по траектории пути; множитель $1 / 2$ перед $\nu_{n}$ отвечает за то, что вершина $i_{t_{1}}$ может оказаться вершиной самопересечения. Легко видеть, что вероятность таких траекторий случайного блуждания экспоненциально убывает с ростом $\nu_{n}$, т.е. существует такая константа const $_{7}$, что доля таких траекторий не превышает

$$
\left(2 s_{n}\right)^{2} e^{-\operatorname{const}_{7} \cdot \nu_{n}}
$$

Пусть $\eta_{n}$ - число ребер, проходимых четыре раза путем с простыми самопересечениями. Тогда $\mathbf{E} \prod_{u=0}^{2 s_{n}-1} \xi_{i_{u} i_{u+1}} \leqslant(1 / 4)^{S_{n}}(8 \text { const })^{2 \eta_{n}}$. При заданном значении $\nu_{n}$ легко написать оценку сверху для числа ребер, проходимых четыре раза, и для величины $(8 \text { const })^{2 \eta_{n}}$. По-прежнему будем обозначать общее число моментов самопересечений через $M$. Как было показано в $\S 4$, достаточно ограничиться случаем $M \leqslant 10 s_{n}^{2} / n$. Для каждого из $M$ моментов самопересечения число возможностей для выбора вершины самопересечения, являющейся концом четырехкратного ребра, не превосходит $\nu_{n}$, в то время как число всех возможностей для выбора вершины самопересечения в общем случае есть величина порядка $s_{n}$. Таким образом, среднее значение $\eta_{n}$ имеет порядок $\left(\nu_{n} / s_{n}\right) M=O\left(\left(\nu_{n} / \sqrt{s_{n}}\right)\left(s_{n}^{3 / 2} / n\right)\right)$. Аналогичные рассуждения показывают, что среднее значение величины $(8 \text { const })^{2 \eta_{n}}$ не превосходит $s_{n}^{1 / 2}(8 \text { const })^{\left(\nu_{n} / \sqrt{s_{n}}\right)\left(20 s_{n}^{3 / 2} / n\right)}$. В результате подсумма суммы $Z_{1}$ по путям с четырехкратными пересечениями и $\nu_{n}>s_{n}^{\gamma}$ ведет себя как

$$
Z_{1} \cdot O\left(\max _{s_{n}^{\gamma}<\nu_{n} \leqslant s_{n}}\left\{(8 \text { const })^{\left(20 s_{n}^{3 / 2} / n\right)\left(\nu_{n} / \sqrt{s_{n}}\right)}\left(2 s_{n}\right)^{2} e^{- \text {const }_{7} \cdot \nu_{n}}\right\}\right)=o\left(Z_{1}\right) .
$$


В случае произвольного четного пути обозначим через $N_{n}$ число неотмеченных моментов времени, при которых выбор способа продолжения траектории («возвращение назад») неоднозначен. В силу определения $N_{n} \leqslant r+\sum_{k=3}^{s_{n}} k n_{k}$. Из рассуждений $\$ 4$ следует, что вероятность выбора вершин пути таким образом, чтобы $N_{n}>N$, есть $O\left(\left(\operatorname{const}_{5} \cdot s_{n} / n^{2 / 3}\right)^{N}\right)$ равномерно по $N$. Указанные $N_{n}$ моментов времени разбивают интервал $\left[0,2 s_{n}\right]$ на $N_{n}+1$ подынтервалов, хотя бы один из которых содержит подподынтервал $\left[t_{1}, t_{2}\right]$, в течение которого траектория как минимум $\nu_{n} /\left(N_{n}+1\right)$ раз снижается до уровня $x\left(t_{1}\right)$, но ни разу не пересекает его. Доля таких траекторий $x(\cdot)$ не превосходит $\left(2 s_{n}\right)^{2} e^{- \text {const }_{7} \cdot \nu_{n} /\left(N_{n}+1\right)}$ (ср. (A1)), и, проводя вычисления, аналогичные использованным в $\S 4$ при подсчете $Z_{1}, Z_{2}$ и $Z_{3}$, получаем, что подсумма по путям, для которых $\nu_{n}>s_{n}^{\gamma}$, ограничена сверху величиной

$$
Z_{1} \cdot\left(\sum_{\nu_{n}=s_{n}^{\gamma}}^{s_{n}} \sum_{N_{n}=0}^{s_{n}}\left(\frac{\text { const }_{5} \cdot s_{n}}{n^{2 / 3}}\right)^{N_{n}} e^{- \text {const }_{7} \cdot \nu_{n} /\left(N_{n}+1\right)}(8 \text { const })^{\frac{\nu_{n}}{\sqrt{s_{n}}} \frac{20 s_{n}^{3 / 2}}{n}}\right) \text {. }
$$

Элементарные вычисления показывают, что второй сомножитель в (А2) есть $o(1)$. Лемма 3 доказана.

Сумма по путям с ребрами кратности четыре и более и $\nu_{n}<s_{n}^{\gamma}$ исследовалась в $\S 4$ (см. оценки для $\left.Z_{1}^{\prime}, Z_{2}^{\prime}\right)$, где было доказано, что эта сумма также есть $o\left(Z_{1}\right)$.

\section{ЛитеРАтУРА}

1. Wigner E. Characteristic vectors of bordered matrices with infinite dimensions. Ann. of Math., 62, 548-564 (1955).

2. Wigner E. On the distribution of the roots of certain symmetric matrices. Ann. of Math. 67, 325-328 (1958).

3. Марченко В. А., Пастур Л. А. Распределение собственных значений в некоторых ансамблях случайных матриц. Матем. сб., 72, 507-536 (1967).

4. Пастур Л. А. О спектре случайных матриц. Теор. матем. физ., 10, 102-112 (1972).

5. Пастур Л. А. Спектры случайных самосопряженных операторов. УМН, 28, 3-64 (1973).

6. Arnold L. On the asymptotic distribution of the eigenvalues of random matrices. J. Math. Anal. Appl., 20, 262-268 (1967).

7. Arnold L. On Wigner's semicircle law for the eigenvalues of random matrices. Z. Wahrscheinlichkeitstheorie Verw. Gebiete, 19, 191-198 (1971).

8. Wachter $K$. W. The strong limits of random matrix spectra for sample matrices of independent elements. Ann. Probab., 6, No. 1, 1-18 (1978).

9. Гирко В. Л. Спектральная теория случайных матриц. Наука, М., 1988.

10. Bai Z. D. Convergence rate of expected spectral distributions of large random matrices. I. Wigner Matrices. Ann. Probab., 21, 625-648 (1993).

11. Khorunzhy A. M., Khoruzhenko B. A., Pastur L. A., Shcherbina M. V. The large $n$-limit in statistical mechanics and the spectral theory of disordered systems. In: Phase Transitions and Critical Phenomena, vol. 15 (Domb C., Lebowitz J. L. eds.), Academic Press, London, 1992.

12. Khorunzhy A. M., Khoruzhenko B. A., Pastur L. A. Asymptotic properties of large random matrices with independent entries. J. Math. Phys., 37, 5033-5059 (1996). 
13. Khorunzhy A. On smoothed density of states for Wigner random matrices. Random Oper. Stochastic Equations, 5, No. 2, 147-162 (1997).

14. Sinai Ya., Soshnikov A. Central limit theorem for traces of large random symmetric matrices with independent matrix elements. Brazilian J. Math., 1998 (в печати).

15. Tracy C., Widom H. On orthogonal and symplectic matrix ensembles. Commun. Math. Phys., 177, 727-754 (1996).

16. Tracy C., Widom H. Level-spacing distribution and the Airy kernel. Commun. Math. Phys., 159, 151-174 (1994).

17. Forrester P. J. The spectrum edge of random matrix ensembles. Nuclear Phys. B, 402, 709-728 (1994).

18. Füredi Z., Komlóz J. The eigenvalues of random symmetric matrices. Combinatorica, 1, No. 3, 233-241 (1981).

19. Буте де Монвель А., Щербина М. В. О норме случайных матриц. Матем. заметки, 57, вып. 5, 688-698 (1995).

20. Mehta M. L. Random Matrices. Acad. Press, New York, 1991.

21. Costin O., Lebowitz J. L. Gaussian fluctuations in random matrices. Phys. Rev. Lett., 75, No. 1, 69-72 (1995).

Princeton University, Dept. of Mathematics

Princeton, NJ, USA

Поступило в редакцию

Institute for Advanced Study,

5 сентября 1997 г.

School of Mathematics, Princeton, NJ, USA 